\title{
Seclusion of molecular layers in a confined simple liquid
}

\author{
Ali Gooneie $\odot,{ }^{1, *}$ Tobias E. Balmer, ${ }^{2, \dagger}$ and Manfred Heuberger $\oplus^{1,2, \ddagger}$ \\ ${ }^{1}$ Laboratory of Advanced Fibers, Empa, Swiss Federal Laboratories for Materials Science and Technology, \\ Lerchenfeldstrasse 5, CH-9014 St. Gallen, Switzerland \\ ${ }^{2}$ Laboratory for Surface Science and Technology, ETH Zürich, Zürich, Switzerland
}

(Received 11 November 2019; revised manuscript received 6 February 2020; accepted 9 April 2020; published 4 May 2020)

\begin{abstract}
We provide theoretical evidence by using molecular dynamics that a nanoconfined film of octamethylcyclotetrasiloxane divides into manifolds of secluded thermodynamic substates. We find equivalence between the splitting into substates and the formation of molecular layers. The seclusion of layers is validated in drainage experiments using an extended surface forces apparatus (eSFA). Furthermore, per-molecule evaluations of the configurational entropy provide evidence for an increased molecular packing upon confinement. The increasing trends in both layer seclusion and molecular packing with confinement explain the exponentially increasing surface forces measured in eSFA.
\end{abstract}

DOI: 10.1103/PhysRevResearch.2.022026

\section{INTRODUCTION}

The behavior of confined liquids is central in many fields including nanotribology, microcontacts, porous materials, and nanofluidics. Despite its significance, the existing research is highly controversial on its physical fundamentals and in particular on the interplay between dynamics, thermodynamics, and molecular configurations. Nanoconfined liquids often have a tendency to organize into layered structures as the film thickness is decreased to a few molecular diameters. For a diversity of liquids, such as globular molecules (e.g., octamethylcyclotetrasiloxane, OMCTS) as well as straightchain alkanes, the layering is portrayed by the oscillatory solvation forces between the confining surfaces [1-8]. It is generally accepted that a nonpolar liquid becomes more solid-like when nanoconfined. This phase transition was initially interpreted as a first-order solidification based on the observation of an abrupt many-order-of-magnitude increase of the effective viscosity of the liquid film upon expulsion of single molecular layers [9]. However, others showed that the changes in the viscosity are continuous and the liquid displays a viscoelastic behavior as if the fluid is approaching a glass transition [10,11]. While these conflicting concepts have fueled a lot of dispute in the field (for some examples, see Refs. [12,13] and the references within), it was also suggested that they are fundamentally coherent and their differences are mainly due to experimental resolution and interpretation [14].

\footnotetext{
${ }^{*}$ Corresponding author: ali.gooneie@empa.ch

${ }^{\dagger}$ Present address: Weibel AG, Bern, Switzerland.

‡Corresponding author: manfred.heuberger@empa.ch
}

Published by the American Physical Society under the terms of the Creative Commons Attribution 4.0 International license. Further distribution of this work must maintain attribution to the author $(s)$ and the published article's title, journal citation, and DOI.
Most of the available knowledge in this field stems from surface forces experiments using surface forces apparatus (SFA) and surface forces balance techniques [15-17]. However, a major drawback of these methods is their inability to resolve the molecular structure directly. Thus, the correlation of a confinement-induced phase transition with the molecular structure of the film as well as its thermodynamic state is still unclear. The existence of molecular layers, however, has been proven experimentally by using x-ray reflectivity $[18,19]$. A less direct approach to study the structure of confined films was developed based on refractive index measurements [8], and was recently invoked to detect density changes that would occur with solidification [12]. The evaluations showed no significant changes in the film density at different confinement levels in comparison with the bulk density and, thus, were argued to rule out any molecular packing that should have been induced by a first-order phase transition. These experimental observations are partially in agreement with molecular simulations that reported an almost constant average fluid density over all thicknesses in spite of density oscillations at layering transitions [20]. Other simulations have shown that the density oscillates through layering transitions and its mean value under confinement is higher than the bulk liquid density [21,22]. The changes in density are directly linked to the altered molecular packing of the film upon confinement and affect the amount of force that the film can bear before it goes through a layering transition. The majority of molecular simulations implement a series of equilibrium simulations between parallel surfaces at fixed film thicknesses [13,23,24]. Recent nonequilibrium molecular simulations of the approach and retraction force cycles have linked force hysteresis to changes in the molecular packing [25]. It is suggested that the energy dissipation between approach and retraction cycles result in less compact films during retraction, thus, lower the measured force. Such structural changes also influence the flow characteristics of confined fluids in squeezing as well as in shearing flows [20,25-29]. Therefore, it is clear 


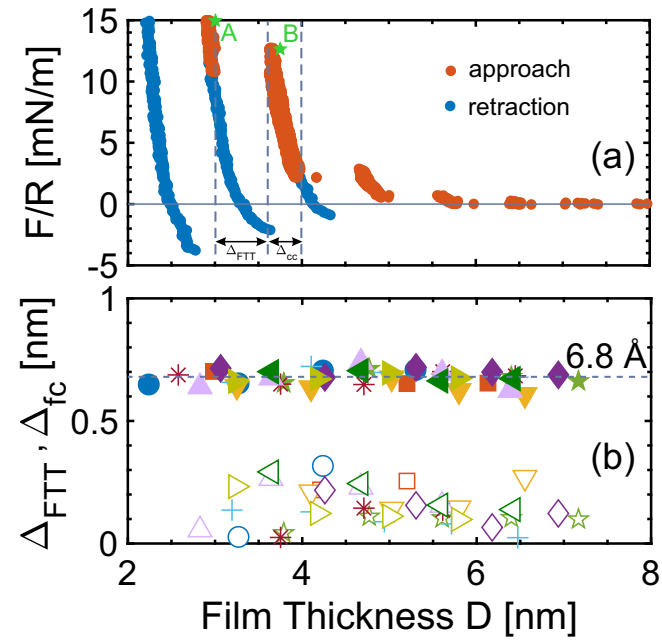

FIG. 1. (a) Oscillatory surface forces in confined OMCTS measured by eSFA. Red points are measured during approach and blue points are measured during retraction succeeding different actuator inversion points. Points A and B correspond to the thicknesses of the parallel sections used in MD simulations. (b) The step size $\Delta_{\text {FTT }}$ (filled symbols) and the net compression of the fluid film $\Delta_{\mathrm{fc}}$ (open symbols) during multiple FTTs. $\Delta_{\mathrm{fc}}$ is calculated from the total contact compression $\Delta_{\mathrm{cc}}$ and is corrected for the optical mica compression. See part (a) and Fig. S3 in the SM [30] for the definitions of these terms. The different symbols in (b) correspond to force measurements from different experiments showing a good reproducibility.

that a fundamental understanding of molecular structures in connection with the thermodynamic state of nanoconfined liquids is required.

To establish the link between layering transitions and the subsequent changes in equilibrium structure and thermodynamics, we combine extended SFA (eSFA) experiments in both quasiequilibrium or nanofluidic situations with molecular dynamics (MD) simulations. Similar to many studies in this field, we take OMCTS as the prototypical nonpolar liquid in our study. Details of eSFA experiments as well as MD simulations are given in the Supplemental Material (SM) [30].

\section{RESULTS AND DISCUSSION}

When OMCTS is confined to nanometer thickness in the eSFA, it exhibits confinement-induced oscillatory surface forces as observed during approach/retraction cycles, Fig. 1(a). Each oscillation represents a layering transition or a film thickness transition (FTT) from $[N]$ to $[N-1]$ layers during force-loaded approach. One can typically observe a sequence of 5-8 FTTs, which suggests a layered molecular configuration in the nanoconfined OMCTS with a characteristic spacing conforming to the molecule size $[18,19]$. Figure 1(b) displays the experimentally measured size of the FTTs, $\Delta_{\text {FTT }}$, which are quasiconstant with a mean value $\Delta_{\text {FTT }}=0.68 \pm$ $0.03 \mathrm{~nm}$. This constant FTT is remarkable in view of the exponential increase of external force acting on the film, indicating the formation of a more densely packed structure. Furthermore, a careful analysis of the film compression segments $\Delta_{\mathrm{fc}}$, which are corrected to account for the optical mica

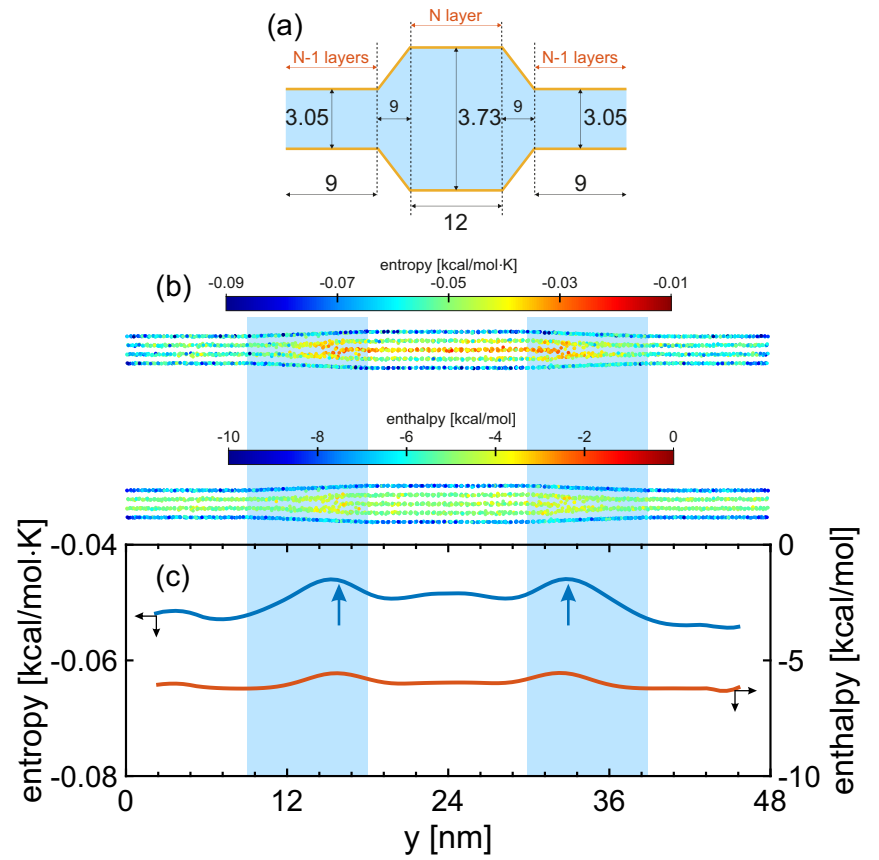

FIG. 2. (a) Schematics of double-wedge geometry and its dimensions used in MD simulations of mica-OMCTS. Note that the simulated depth of the wedges was constant everywhere and set to $9 \mathrm{~nm}$. (b) Layered configuration of OMCTS molecules at equilibrium from MD. Each molecule is colored according to its per-molecule variables of state, once by its entropy and once by its enthalpy. (c) Lateral averages of entropy and enthalpy across the film (bin size $0.5 \mathrm{~nm}$ ). The mean entropy exhibits discernable peaks in the wedged sections close to the layering transitions where the central layers merge in part (b) (arrows). The intensity of the entropy peaks is influenced by the slope of the wedge and the wall-liquid interactions (see Fig. S5 in the SM [30]). The average entropy is higher in the thicker portions of the parallel film indicating a less ordered structure.

compression (for details, see SM [30]), reveals a mean value $\Delta_{\mathrm{fc}}=0.14 \pm 0.08 \mathrm{~nm}$, which is also remarkably constant. Thus, each expelled layer accounts for an approximate change in film thickness of $\Delta_{\mathrm{FTT}}+\Delta_{\mathrm{fc}}=0.82 \mathrm{~nm}$. Consequently, for the film to be able to bear exponentially increasing forces during subsequent FTTs either the internal structure of the layers or the correlations between them must have changed (or a combination of the former and the latter).

Considering the equivalence between the oscillatory surface potential and combined contributions of entropy and internal energy [20], as well as density changes [31], MD simulations are incorporated to reveal layer-specific structures and thermodynamic characteristics. MD simulations were performed in a wedge geometry schematically shown in Fig. 2(a). This geometry imposes a layering transition at equilibrium that simultaneously coexists with its parent well-formed (parallel) layered configurations. This is in contrast to previous simulations considering nonequilibrium transitions induced by moving surfaces, or equilibrium models with fixed parallel surfaces [31]. In this way, we can study the structure of films before, during and after a layering transition without any contributions of the nonequilibrium energy dissipation 
to fluid structure [21]. Furthermore, we link the molecular structures directly to per molecule configurational entropy by using the two-body approximation of the Ornstein-Zernike equation $[32,33]$. The summation of intermolecular potentials serves to calculate the local enthalpy $[30,34,35]$. Relative to the bulk liquid state (see Fig. S4 in the SM [30]), our evaluations revealed downshifts in both entropy and enthalpy in the nanoconfined film. Remarkably, the color-coded OMCTS molecules in Fig. 2(b) reveal that individual layers correspond to distinctive thermodynamic substates. In this paper, a substate refers to the thermodynamic state of an individual molecular layer, which is itself a subset of the cumulative thermodynamic state of the entire liquid manifold in one of the parallel sections of the simulated geometry. We note that the layers adjacent to the mica walls exhibit particularly strong downshifts. A similar yet smoother gradient in the thermodynamic states of confined liquids is also reported for water confined inside a carbon nanotube [36]. Interestingly, the liquid shows a local increase in the mean entropy midway into the wedge segments close to the layering transition, i.e., where the layers merge [arrows in Fig. 2(c)]. This local increase in the entropy, i.e., loss of molecular ordering, in the central layers entails enhanced interlayer diffusion, enabling layer merging during an FTT $[20,31]$ in agreement with previous nonequilibrium simulations [25] and drainage experiments [27]. The merging of central layers, which is responsible for the compression of the film between layering transitions, could explain the constant $\Delta_{\text {FTT }}$.

For an in-depth analysis of the layering structures in parallel sections, it is instructive to plot per-molecule thermodynamic variables in a state diagram (Fig. S7 in the SM [30]), where each OMCTS molecule represents a point with a layerspecific color code, according to the illustration in Fig. 3(a). The mean thermodynamic properties of the layers (substates) are plotted as colored rectangular symbols in Fig. 3(a) by averaging the enthalpy and entropy of all molecules that belong to a specific layer. Individual layers unambiguously define distinctive substates. These individual substates together form the $[N]$ manifold of an equilibrium film with $\mathrm{N}$ layers. For convenience, we choose to denote substates as $\sigma_{[i, N]}$ where $\mathrm{i}$ indicates the individual layer(s) in the $[N]$ manifold counting away from the walls. The entire $[N]$-manifold of substates is labeled $\Sigma_{[N]}$. The OMCTS molecules from the unconfined bulk liquid simulation (Fig. S4 in the SM [30]) are added as bulk reference state (green) to Fig. 3(a). For reference, we have also calculated the entropy of the bulk three-dimensional (3D)-crystal OMCTS [37], and marked it as horizontal dotted line in the state diagram. Remarkably, different molecular layers correspond to secluded substates of the manifolds that fall between the bulk liquid and solid references. The density profiles of $\Sigma_{[5]}$ and $\Sigma_{[4]}$ manifolds are plotted in Fig. 3(b). The distinct density peaks indicate individual molecular layers. Individual layers are secluded since there is negligible probability of OMCTS molecules residing between the layers. The effective potential of an OMCTS molecule as part of the layered structures can be estimated from the Boltzmann relation (see Fig. S6b in the SM [30]). The layered structure thus implies energy barriers between the molecular layers that are much larger than $k_{B} T$. This translates into an activated interlayer diffusion of molecules in
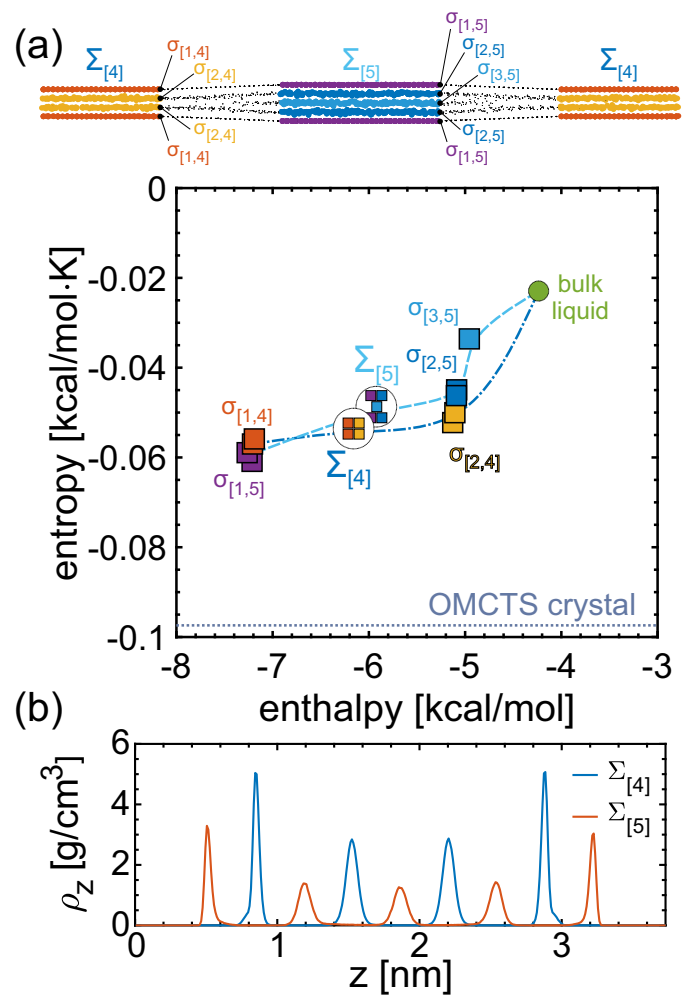

FIG. 3. (a) Thermodynamic state diagram analysis of molecules in the two parallel sections of the wedge geometry; (top) illustration of layer-specific color scheme and indexing convention introduced to identify individual substates (layers) and their manifolds; (bottom) condensed form of the state diagram marking the mean substate loci by filled squares $\sigma_{[n, N]}$ (representing each individual layer), as well as the cumulative loci of the whole films shown by white circles $\Sigma_{[N]}$ (representing each parallel section containing multiple layers). The state diagram displaying every molecule in its layer-specific color is shown in Fig. S7a in the SM [30]. (b) The strong modulation of the density profile across the film $\rho_{z}$ in the $\Sigma_{[4]}$ and $\Sigma_{[5]}$ film states indicate secluded molecular layers.

the layered film [20,38,39]. An effective activation barrier for interlayer diffusion is indeed seen experimentally as a critical loading force [Fig. 1(a)] required to push molecular layers into each other and nucleate an FTT. The strong increase of the diffusion energy barriers upon reduction of film thickness readily explains the exponential increase of FTT peak surface force. Also, the average entropy of the nanoconfined films decreases with diminishing thickness, which is a clear indication of the development of ordered structures with higher molecular packing. This trend clearly suggests an increasing overall film density upon confinement. Hence, a direct link is established between the molecular structures, thermodynamics, seclusion of layers, and mechanical response seen as oscillatory forces.

An integral thermodynamic description of the $\Sigma_{[N]}$ manifolds emerges from averaging entropy and enthalpy of all molecules in the film. These manifold state variables are shown in Fig. 3(a) by white circles that contain rectangles symbolizing constituting substates. The $\Sigma_{[4]}$ and $\Sigma_{[5]}$ loci suggest a gradual transition from the unconfined bulk liquid towards the 3D solid crystal by increasing confinement. These calculations also show how profoundly the thermodynamic 

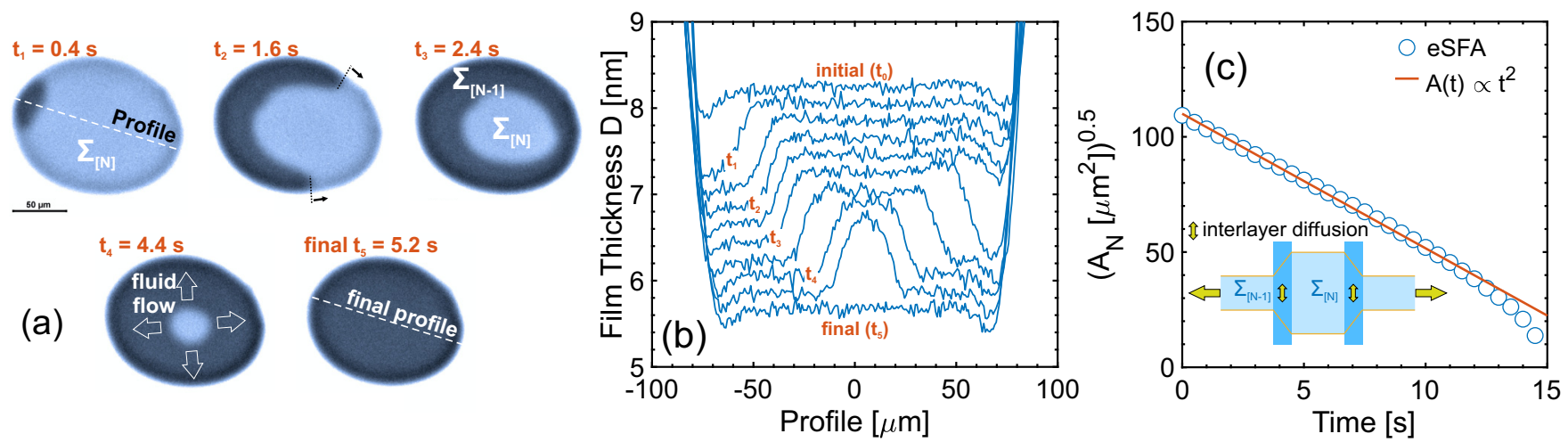

FIG. 4. (a) Sequence of OSC images recorded during the liquid drainage experiments. The FTT was nucleated at the perimeter, then a $\Sigma_{[N]}$ island (bright blue) was trapped and continuously drained into a sea of $\Sigma_{[N-1]}$ (dark blue). (b) Stacked illustration of film thickness profiles during layered island drainage experiments from $[N]$ to $[N-1]$ layers, measured at different times $t_{0}$ to $t_{5}$ corresponding to the OSC images shown in part (a). The final profile is the measured film thickness and the preceding profiles are graphically offset by $0.2 \mathrm{~nm}$ for better visibility. Based on OMCTS molecular size and simulations $N$ equals eight layers. (c) The changes in the square root of island area $\sqrt{A_{N}(t)}$ over time suggest that interlayer diffusion, which limits the drainage rate, is predominantly occurring at the island perimeter. Note that the drainage rate is slower than that in parts (a), (b) for more accurate measurement of the island area.

fingerprints of the nanoconfined 2D layered films differ from both solid and liquid reference states of OMCTS. Such substate analysis in a state diagram provides a means to study how confined films can split into layers with distinct thermodynamic states that unfold between 3D "solid" and "liquid" and thus exhibit ostensibly contradicting properties. The intermediate thermodynamic manifold states of the nanoconfined films are similar to a continuous solidification [10] or glass transition, if the underlying substate splitting is overlooked.

The predicted layer seclusion and the layer-specific entropy should have assessable effects on the nanofluidics and FTT propagation. We experimentally measured the kinetics of an island of $[N]$ layers draining into a sea of $[N-1]$ layers using the eSFA. The procedures used for generating such islands in the eSFA are described in the SM [30]. Changes of film thickness were recorded in real-time by a monochrome CCD camera and evaluated using the optical spectral correlation (OSC) method, which analyzes film thickness across an optical correlator by record of photometric intensities [40]. An animated movie of a representative fluidic experiment is also available in the SM [30]. The idea of this experiment is to record the area of an island in state $\Sigma_{[N]}$, surrounded by a film in state $\Sigma_{[N-1]}$. Figure 4(a) illustrates a sequence of typical OSC snapshots. Different intensities correspond to different number of layers in the fluid film. We note that the island retains its secluded $[N]$ layered structure during the extent of the drainage experiments. Otherwise, we should have seen a gradual decrease of the island thickness [see Fig. 4(b)] in terms of change of the gray level. If the molecules could easily permeate between the layers, the layered structure would not persist in the island and the film would not undergo stepwise thickness changes as observed in the snapshots (and the movie in the SM). The temporal evolution of the area of the completely isolated island, $A_{N}(t)$ is recorded and shown in Fig. 4(c). If the drainage rate of the layered island is determined by interlayer diffusion at the perimeter (ring wedge), then one expects $d A / d t \propto \sqrt{A}$ leading to $A(t) \propto t^{2}$ progression. Comparison of the fitted data in Fig. 4(c) shows that the perimeter controls the drainage rate, which is in agreement with previous measurements [27]. This observation shows that the permeation of molecules between layers mainly occurs in a well-defined peripheral region (while the island retains its layered structure), and propagates by a front-dynamics to complete a layering transition. Although this simple drainage experiment cannot resolve all molecular details, it shows that interlayer diffusion mostly occurs in the wedge, which is in agreement with the entropy peaks seen in the MD simulations [Figs. 2(b) and 2(c)]. Previous MD simulations have also documented noticeable changes in the overall entropy of the film during the layering transitions [20]. Our results suggest that only the central layer(s) participate in molecular rearrangements during a layering transition. The structural changes, from a well-formed layered configuration to centrally disordered, to a new layered state, are well manifested by the configurational entropy. We note that the fluid-wall interaction is a key factor that drives substate splitting. The state diagram in Fig. 3(a) illustrates that the middle layer, $\sigma_{[3,5]}$, performs a thermodynamically favorable enthalpy downshift during the $\Sigma_{[5]}$ to $\Sigma_{[4]}$ transition. At the same time, it displays a thermodynamically unfavorable entropy downshift. Thus, the enthalpy of adsorption must be considered a key design parameter in nanofluidics. In support of this hypothesis, we repeated the MD simulations for stronger fluid-wall interactions and found that the substate splitting and seclusion were both amplified (Figs. S5, S6a, and S7d-S7f in the SM [30]). Additionally, the potential for implementation of substate splitting in a nanofluidic device opens the interesting possibility of controlling interlayer diffusion and flow in the center of a nanofluidic channel via subnanometer adjustments of channel width and/or tuning nanochannel wall composition $[29,39,41,42]$.

\section{CONCLUDING REMARKS}

In summary, using MD simulations and eSFA experiments of OMCTS, we found that the nanoconfined films in parallel 
channels split into individual molecular layers corresponding to thermodynamic substates. These substates become more secluded with increasing confinement, and effective activation barriers prevent interlayer diffusion. The layer seclusion as well as the molecular packing in the layers increase upon confinement. This demonstrates the correlation between the exponentially increasing surface forces and the molecular structure of the films.

\section{ACKNOWLEDGMENTS}

We acknowledge scientific discussions with Dr. E. Perret and Dr. R. Hufenus (both Empa), and Prof. K. G. Ayappa and Dr. V. Vadhana (both at the Indian Institute of Science). During this research, A.G. received support from the Empa-Internal research Call 2018/project "ALLFIN". The contribution of T.E.B. was possible thanks to the Swiss National Funds project SNF 200020-107452.
[1] R. G. Horn and J. N. Israelachvili, Chem. Phys. Lett. 71, 192 (1980).

[2] H. K. Christenson, J. Chem. Phys. 78, 6906 (1983).

[3] H. K. Christenson, J. Phys. Chem. 90, 4 (1986).

[4] R. M. Espinosa-Marzal, T. Drobek, T. Balmer, and M. P. Heuberger, Phys. Chem. Chem. Phys. 14, 6085 (2012).

[5] M. P. Heuberger, Z. Zachariah, N. D. Spencer, and R. M. Espinosa-Marzal, Phys. Chem. Chem. Phys. 19, 13462 (2017).

[6] Z. Zachariah, R. M. Espinosa-Marzal, N. D. Spencer, and M. P. Heuberger, Phys. Chem. Chem. Phys. 18, 24417 (2016).

[7] P. Richetti and P. Kékicheff, Phys. Rev. Lett. 68, 1951 (1992).

[8] M. Heuberger, M. Zäch, and N. D. Spencer, Science 292, 905 (2001).

[9] J. Klein and E. Kumacheva, Science 269, 816 (1995).

[10] A. L. Demirel and S. Granick, Phys. Rev. Lett. 77, 2261 (1996).

[11] P. A. Thompson, G. S. Grest, and M. O. Robbins, Phys. Rev. Lett. 68, 3448 (1992).

[12] D. F. Kienle and T. L. Kuhl, Phys. Rev. Lett. 117, 036101 (2016).

[13] P. T. Cummings, H. Docherty, C. R. Iacovella, and J. K. Singh, AIChE J. 56, 842 (2010).

[14] A. L. Demirel and S. Granick, J. Chem. Phys. 115, 1498 (2001).

[15] D. Tabor and R. H. S. Winterton, Nature (London) 219, 1120 (1968).

[16] J. N. Israelachvili and D. Tabor, Nat. Phys. Sci. 236, 106 (1972).

[17] J. Israelachvili, Y. Min, M. Akbulut, A. Alig, G. Carver, W. Greene, K. Kristiansen, E. Meyer, N. Pesika, K. Rosenberg, and H. Zeng, Rep. Prog. Phys. 73, 036601 (2010).

[18] E. Perret, K. Nygård, D. K. Satapathy, T. E. Balmer, O. Bunk, M. Heuberger, and J. F. van der Veen, EPL 88, 36004 (2009).

[19] E. Perret, K. Nygård, D. K. Satapathy, T. E. Balmer, O. Bunk, M. Heuberger, and J. F. van der Veen, J. Phys.: Condens. Matter 22, 235102 (2010).

[20] J. Gao, W. D. Luedtke, and U. Landman, Phys. Rev. Lett. 79, 705 (1997)

[21] Y. Leng, Y. Xiang, Y. Lei, and Q. Rao, J. Chem. Phys. 139, 074704 (2013).
[22] S. T. Cui, P. T. Cummings, and H. D. Cochran, J. Chem. Phys. 114, 7189 (2001).

[23] V. Vadhana and K. G. Ayappa, J. Phys. Chem. B 120, 2951 (2016).

[24] K. G. Ayappa and R. K. Mishra, J. Phys. Chem. B 111, 14299 (2007).

[25] R.-G. Xu and Y. Leng, Proc. Natl. Acad. Sci. USA 115, 6560 (2018).

[26] T. Becker and F. Mugele, Phys. Rev. Lett. 91, 166104 (2003).

[27] L. Bureau, Phys. Rev. Lett. 104, 218302 (2010).

[28] Y. Lei and Y. Leng, Phys. Rev. Lett. 107, 147801 (2011).

[29] K. Wu, Z. Chen, J. Li, Z. Lei, J. Xu, K. Wang, R. Li, X. Dong, Y. Peng, S. Yang, F. Zhang, Z. Chen, and Y. Gao, J. Phys. Chem. C 123, 16456 (2019).

[30] See Supplemental Material at http://link.aps.org/supplemental/ 10.1103/PhysRevResearch.2.022026 for simulation details, experimental methods, and complementary results.

[31] L. G. Cámara and F. Bresme, J. Chem. Phys. 120, 11355 (2004).

[32] D. Henderson, F. F. Abraham, and J. A. Barker, Mol. Phys. 31, 1291 (1976).

[33] F. F. Abraham, J. Chem. Phys. 68, 3713 (1978)

[34] P. M. Piaggi, O. Valsson, and M. Parrinello, Phys. Rev. Lett. 119, 015701 (2017).

[35] P. M. Piaggi and M. Parrinello, J. Chem. Phys. 147, 114112 (2017).

[36] A. Barati Farimani and N. R. Aluru, J. Phys. Chem. C 120, 23763 (2016).

[37] H. Steinfink, B. Post, and I. Fankuchen, Acta Crystallogr. 8, 420 (1955).

[38] H. Matsubara, F. Pichierri, and K. Kurihara, Phys. Rev. Lett. 109, 197801 (2012).

[39] G. J. Wang and N. G. Hadjiconstantinou, Langmuir 34, 6976 (2018).

[40] T. E. Balmer and M. Heuberger, Rev. Sci. Instrum. 78, 093105 (2007).

[41] G. J. Wang and N. G. Hadjiconstantinou, Phys. Rev. Fluids 4, 064201 (2019).

[42] M.-D. Krass, G. Krämer, U. Dellwo, and R. Bennewitz, Tribol. Lett. 66, 87 (2018). 